\title{
CORESHINE : a tracer of grain growth in dark clouds
}

\author{
Laurent Pagani $^{* a}$, Charlène Lefèvre ${ }^{a}$, Roberta Paladini ${ }^{b}$, Jürgen Steinacker ${ }^{c}$, Aurore \\ Bacmann $^{c}$, Thomas Henning ${ }^{d}$, and the Spitzer Cycle 8 Hunting Coreshine with Spitzer \\ team \\ ${ }^{a}$ LERMA, UMR 8112, CNRS E Observatoire de Paris, France \\ ${ }^{b} I P A C$, Caltech, Pasadena, USA \\ ${ }^{c} I P A G$, Grenoble, France \\ ${ }^{d}$ MPIA, Heidelberg, Germany \\ E-mail: laurent.pagani@obspm.fr, charlene.lefevre@obspm.fr, \\ paladini@ipac.caltech.edu, stein@mpia.de, \\ aurore.bacmann@obs.ujf-grenoble.fr, henning@mpia.de
}

\begin{abstract}
Scattering by dust grains in the interstellar medium is a well-known phenomenon in the optical and near-infrared domains. We serendipitously discovered the effect of scattering in the midinfrared in the dark cloud L183, and nicknamed the effect "coreshine". We investigated over 200 sources from both the Spitzer Archive and a new warm Spitzer mission program to check the frequency of the phenomenon and found over $50 \%$ of the cases to be positive, which is possibly only a lower limit. We see differences depending on the Galactic regions we investigate. Taurus is a highly successful target while the Galactic plane is too bright to let coreshine appear in emission. We present coreshine as a large grain tracer and we discuss its absence in the Gum/Vela region, which would indicate that big grains have been recently destroyed by the supernova blast wave. Finally, we discuss the prospect for future coreshine searches from archives, present and future instruments.
\end{abstract}

The Life Cycle of Dust in the Universe: Observations, Theory, and Laboratory Experiments 18-22 November, 2013

Taipei, Taiwan

\footnotetext{
* Speaker.
} 


\section{Introduction}

Stars form in cold dark clouds and to understand this formation process, the first step is to understand the physics of these molecular clouds. We want to know how the clouds locally build up to form dense cores $\left(n\left(\mathrm{H}_{2}\right) \sim 10^{4}-10^{5} \mathrm{~cm}^{-3}\right)$, how some of them continue to grow in density and mass to form prestellar cores $\left(n\left(\mathrm{H}_{2}\right) \geq 10^{5} \mathrm{~cm}^{-3}\right.$, [1] $)$ and how they collapse to form protostars. Is contraction due to supersonic turbulence fast decay $[2,3]$ or is it due to quasi-static contraction such as that driven by slow ambipolar diffusion in a magnetically subcritical cloud [4-6] or a combination of turbulence and ambipolar diffusion [7, 8]? How do depletion, surface chemical reactions and desorption of gaseous volatile components depend on the grains, and on UV and cosmic rays penetration? To make progress, we need to collect data from these clouds but this has been a difficult task for a long time until the first submillimeter bolometers became available. Only with the advent of such instruments could one detect the deeply buried cold dust condensations since neither the optical nor the near-infrared (NIR) cameras could peer inside the clouds beyond a few $A_{V}$ in optical and $A_{V} \sim 20$ mag in the $K$ band [9]. The first detections, obtained with a single pixel bolometer [10] opened a new era in the investigation of prestellar cores. However, the spatial resolution of these cameras (e.g. 11" for MAMBO on the IRAM-30m at a wavelength of $1.1 \mathrm{~mm}$, $14^{\prime \prime}$ for SCUBA on the JCMT at $\lambda=850 \mu \mathrm{m}$ ) is slightly too low to explore the details of the cores and these cores are usually too large and smooth to be mapped with interferometers. The situation recently changed with the discovery of a phenomenon that we have named coreshine.

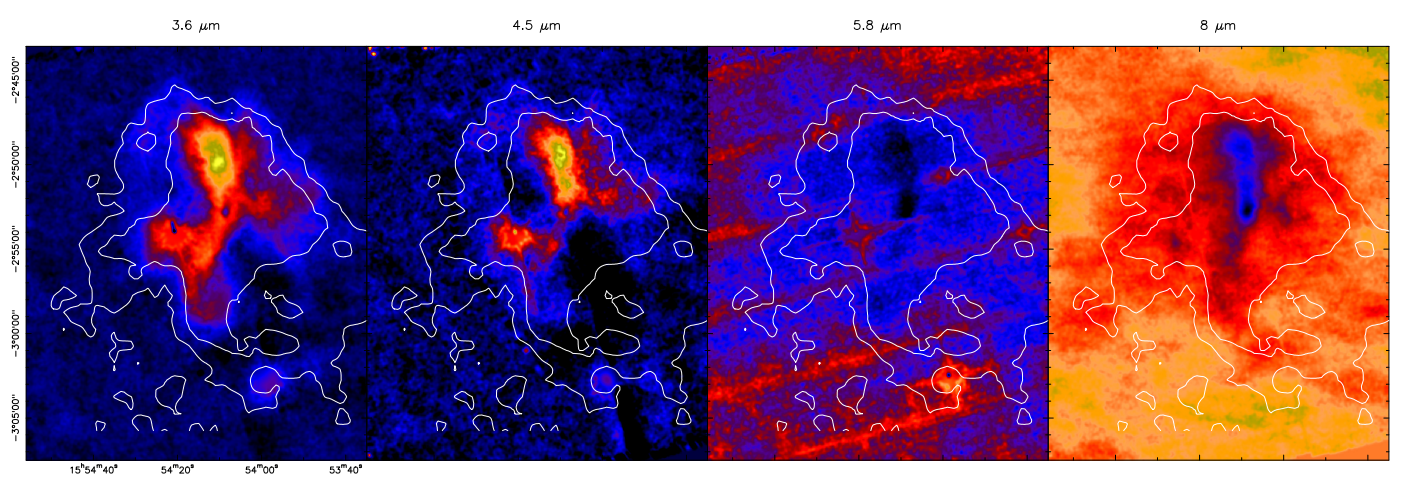

Figure 1: The L183 region as seen by Spitzer/IRAC instrument. Stars and background have been removed following prescriptions of Lefèvre et al. [11]. Contours represent $A_{V}=5$ and 10 mag limits (from [12])

It was serendipitously that we discovered a mid-infrared (MIR) signal in emission in L183 from the Spitzer/IRAC bands $1(3.6 \mu \mathrm{m})$ and $2(4.5 \mu \mathrm{m})$ in coincidence with the peak of the silicate band absorption seen in the Spitzer/IRAC band 4 ( $8 \mu \mathrm{m}$, Fig. 1). It became rapidly obvious that this emission could not come neither from Polycyclic Aromatic Hydrocarbons (PAHs) nor from hot grains. PAHs are not emitting in the IRAC band 2 but are expected to be bright in bands 3 $(5.8 \mu \mathrm{m})$ and $4(8 \mu \mathrm{m})$ whereas the L183 images in these two bands show absorption (Fig. 1). Hot grains are not expected either since the emission is clearly originating from deep inside the cloud, underlying the long cold and dense filament revealed in the previous studies of the source [12 - 14]. Being left with the only possible hypothesis of efficient scattering by dust grains and since MRNtype grains [15] could not be the solution, we came with the conclusion that grown grains were 
needed. Indeed, the scattering coefficient increases faster and becomes larger than the absorption coefficient for grains reaching a size comparable to the photon wavelength, favoring the emergence of the scattered signal. We ran a 3D model to prove the concept [16]. The model was working with a single grain size averaged over the grain size distribution and we made the average grain size grow from $50 \mathrm{~nm}$ at the edge of the cloud to $\sim 1 \mu \mathrm{m}$ in the densest parts as a simple function of the density. We managed to reproduce the large scale features of the $3.6 \mu \mathrm{m}$ observations including the self-absorbed peak due to the prestellar core (characterized by a peak density $\geq 10^{6} \mathrm{~cm}^{-3}$, and a column density $\geq 10^{23} \mathrm{~cm}^{-2},[12]$ ). This was not the first study to invoke grain growth (e.g. [17-19]) but it was the first one which could directly trace micron-size grains. In a second step, we searched the Spitzer archive to find if L183 was a fluke and out of 110 examined fields we found over $50 \%$ cases of coreshine [20]. The study revealed that sources too close to the Galactic plane were never seen in emission and we attributed this effect to a contrast problem : the attenuation of a strong background cannot be compensated by the increase of scattering due to large grains. For all those clouds away from the Galactic plane that present coreshine emission, we now have a tool to explore the depths of the clouds with a resolution ( $\left.\sim 1^{\prime \prime}\right)$ and a coverage (a few arcminutes) which are suited to the dense core sizes.

\section{Recent results}

\subsection{Gum/Vela region}

Among the studied regions in the Spitzer Archive survey, all those outside the Galactic plane were showing at least $50 \%$ of positive cases except two, the Gum/Vela region, which displayed only negative ones, and Orion, which is dominated by a bright background and strong PAH emission and thus not suitable to search for coreshine cases. We suspected the Gum/Vela region to have been cleared of all grown grains by one or several supernovae which are located there, the region being conspicuous in X- and gamma-ray emissions (shocked gas and Vela pulsar). To check this hypothesis, in a subsequent study, we collected all the clouds observed around the Gum/Vela region from the Spitzer Archive, and found 24 fields that could be used. The statistics were indeed quite different from those of the rest of the Galaxy, with only 3 coreshine detections and 3 possible detections ( 2 of them being outside the Gum region, at the edge), with 5 out of 6 containing a Class 0, Class I or IRAS source (the presence of a nearby protostar can partly compensate for the grain scattering efficiency in case of smaller grains). This represents $16 \%$ of coreshine cases inside the Gum region compared to $\sim 50 \%$ for the general survey. We also find 9 bright rimmed clouds or with PAHs out of $24(38 \%)$, while such clouds are less than $10 \%$ in the [20] survey and 7-8 times less frequent than coreshine cases. Finally, though most clouds of the Gum/Vela region have a protostar inside or very close to them, they display no coreshine. It was therefore concluded that the general absence of coreshine and presence of PAHs emission was compatible with the possibility that the whole Gum region be due to one or several supernovae. Indeed, the region centre is void of dark clouds and the outer parts filled with clouds without big grains but having recently formed protostars, and with PAHs probably eroded from the grains (e.g., [21]). The clouds at the periphery of the supernova remnant having been reached only recently by the blast wave have had no time to rebuild big grains yet. 


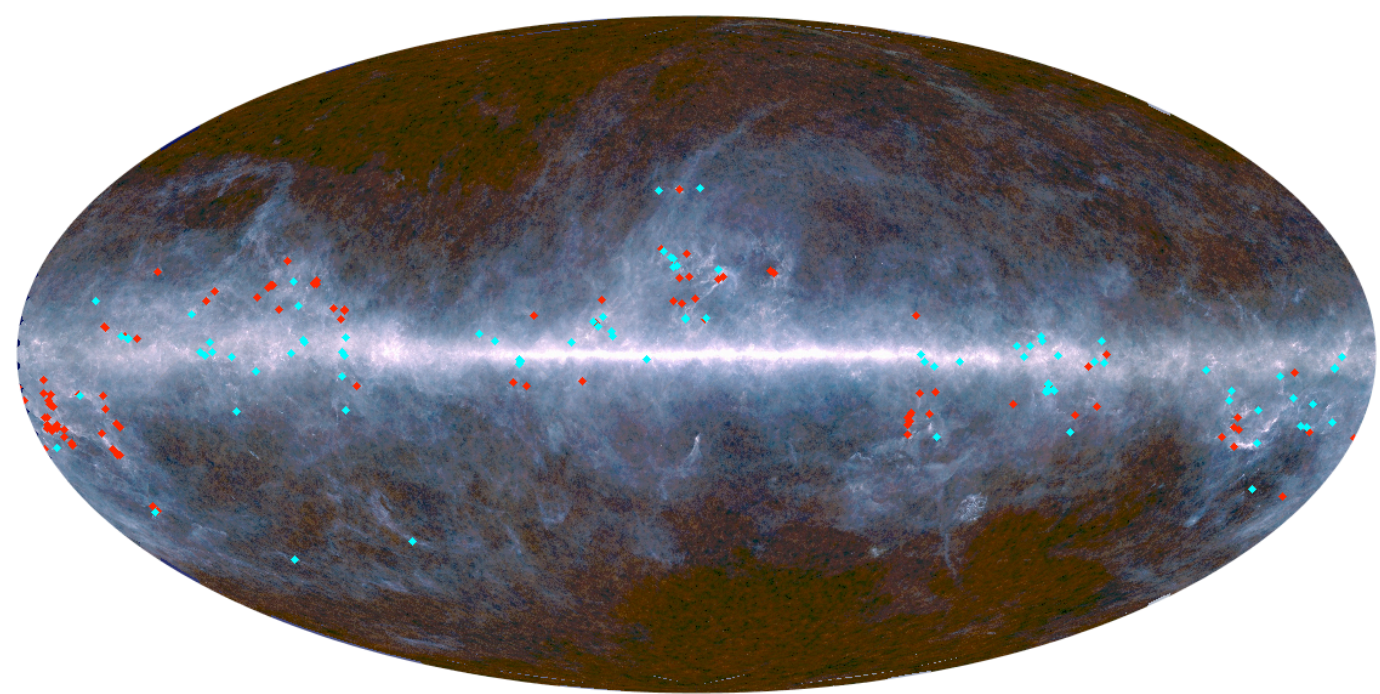

Figure 2: Mollweide map of the repartition of the coreshine cases across the Galaxy (Planck-HFI 353-545$857 \mathrm{GHz} 3$ color image). Red diamonds are coreshine detections, blue ones are negative cases (absorption, PAHs, or nothing visible).

This is one example of the use of coreshine (or its absence) to help trace the evolution of a region. L1506C [18, 22] is another intriguing case of cloud evolution where coreshine could bring some clues though a convincing solution remains to be found [23].

\subsection{Warm Spitzer survey}

Since the [20] survey was based on a selection of clouds taken mostly from 3 programs [2426], its homogeneity was questioned because the exposure times of the different programs were different and the selection of sources possibly biased. It was decided to launch a new survey [27] dedicated to the search of coreshine in cold cores issued from the Planck Early Cold Core (ECC) catalogue $[28,29]$. This survey included 90 cores from the 915 cores of the catalogue chosen in order to cover a range of cases (distance, mass, position in the Galaxy,...). Merged with the previous survey and including a number of corrections (misidentifications) we still find an overall coreshine detection rate of $\sim 50 \%$ (115 cases out of 213,14 of them being tentative, Fig. 2).

\section{Future prospects}

\subsection{Modelling}

Without modelling, the coreshine effect would remain simply the most direct proof of the existence of grown grains in clouds, but coreshine can be used as a tool to explore both the 3D structure of clouds and the grain properties [11,30,31]. Several questions arise to whether a constant amount of large grains is present throughout the cloud or if the large grain fraction increases with density, the importance of the local interstellar radiation field, of the global interstellar radiation field anisotropy and of the cloud background field. The latter can prevent the detection of coreshine 
if too high, as already advocated in [20] and demonstrated in [30,31]. The precise estimate of the background field is a compulsory requisite to be able to constrain the coreshine intensity in the model. This is a very difficult task which must not be underestimated [11]. Eventually, all bands, from visible to submm, should be included in a single model of a dark cloud to try to reproduce absorption, scattering and emission by grains. Coreshine brings here a substantial improvement upon previous works because it strongly helps to characterize the 3D structure of the cloud and the grain size distribution. Such a complete model should be able to constrain both the cloud and the grain properties.

\subsection{Observations}

How can we observe coreshine presently? The Spitzer Archive remains at the moment the best place where to look for coreshine in dark clouds. Both the cold and warm missions are useful. In the case of the warm mission (see e.g [27, 32]), the WISE [33] band $3(12 \mu \mathrm{m})$ data have to be checked to be sure that the emission does not come from PAHs and that the peak of absorption coincides with the signal seen at both 3.6 and $4.5 \mu \mathrm{m}$. NIR scattering (sometimes refered to as cloudshine) is also a possible means of core investigation but only for low opacity cores $\left(A_{V} \leq 20\right.$ mag, [9, 34]). The advantage here is that the observations can be performed from the ground.

WISE itself could be useful since it contains the whole sky but the lack of sensitivity and the large instrumental PSF (6") prevent the detection of most cases except the brightest ones like L183, L260 or L134. In [35], they checked the coreshine presence for 55 ECC cores which included the L183 and L134 known cases and had a very low rate of detection $(\sim 10 \%)$. It can be noticed that the L134 core Spitzer map was too small to reach the edges of the cloud and it was reported as a negative case by absence of contrast in the [20] on-line table while in WISE channels 1 to 3, it is clearly seen.

Akari [36] might be another useful past space mission for coreshine searches in its archive. Presently, the calibrated and reduced data are not yet available to evaluate its potential but a handful of cores have been found to show coreshine (G. Kim, private comm.).

Only SOFIA, an airborne stratospheric observatory [37] is presently equipped with a NIRMIR camera (FLITCAM) in the $1-5 \mu \mathrm{m}$ range, but its sensitivity seems to be $\sim 70$ times lower than Spitzer/IRAC 1. As exposures of several minutes were needed with IRAC to detect coreshine, this would translate to hundreds of hours with SOFIA to observe a single cloud.

JWST is the next space mission. It will be equipped with extremely sensitive NIR and MIR cameras but their field of view will be small and not suited to map extended clouds. They could be used to study coreshine cases in remote clouds to extend the study beyond the Gould Belt survey but these clouds will be inside the Galactic plane and therefore seen in absorption only. They might also be used to search for coreshine effect in a few nearby galaxies (Magellanic clouds, M33, or possibly M31), provided that the galaxy is facing us enough to have a low background.

Finally, a recently proposed JAXA mission, named $\mathrm{WISH}^{1}$, though mostly devoted to high redshift studies would be a fantastic machine to record coreshine emission on hundreds of square degrees thanks to its wide field cameras covering 5 bands from 1 to $5 \mu \mathrm{m}$, thus covering both NIR and MIR scattering by dark clouds.

\footnotetext{
${ }^{1}$ http://wishmission.org/en/index.html
} 


\section{References}

[1] Keto, E. \& Caselli, P. 2008, ApJ, 683, 238

[2] Klessen, R. S., Heitsch, F., \& Mac Low, M.-M. 2000, ApJ, 535, 887

[3] Larson, R. B. 2007, Reports on Progress in Physics, 70, 337

[4] Mouschovias, T. C. 1991, ApJ, 373, 169

[5] Ciolek, G. E. \& Basu, S. 2000, ApJ, 529, 925

[6] Ciolek, G. E. \& Basu, S. 2006, ApJ, 652, 442

[7] Adams, F. C. \& Shu, F. H. 2007, ApJ, 671, 497

[8] Adams, F. C. 2009, Magnetic Fields in the Universe II: From Laboratory and Stars to the Primordial Universe (Eds. A. Esquivel), 36, 73

[9] Juvela, M., Pelkonen, V.-M., Padoan, P., \& Mattila, K. 2006, A\&A, 457, 877

[10] Ward-Thompson, D., Scott, P. F., Hills, R. E., \& André, P. 1994, MNRAS, 268, 276

[11] Lefèvre, C., Pagani, L., Juvela, M. et al., A\&A, submitted

[12] Pagani, L., Bacmann, A., Motte, F., et al. 2004, A\&A, 417, 605

[13] Pagani, L., Lagache, G., Bacmann, A., et al. 2003, A\&A, 406, L59

[14] Pagani, L., Daniel, F., \& Dubernet, M.-L. 2009, A\&A, 494, 719

[15] Mathis, J. S., Mezger, P. G., \& Panagia, N. 1983, A\&A, 128, 212

[16] Steinacker, J., Pagani, L., Bacmann, A., \& Guieu, S. 2010, A\&A, 511, A9

[17] Ossenkopf, V., \& Henning, T., 1994, A\&A, 291, 943

[18] Stepnik, B., Abergel, A., Bernard, J.-P., et al. 2003, A\&A, 398, 551

[19] Ormel, C. W., Paszun, D., Dominik, C., \& Tielens, A. G. G. M., 2009, A\&A, 502, 845

[20] Pagani, L., Steinacker, J., Bacmann, A., Stutz, A., \& Henning, T. 2010, Science, 329, 1622

[21] Andersen, M., Rho, J., Reach, W. T., Hewitt, J. W., \& Bernard, J. P. 2011, ApJ, 742, 7

[22] Pagani, L., Ristorcelli, I., Boudet, N., et al. 2010, A\&A, 512, A3

[23] Steinacker, J., Ormel, C., Andersen, M., et al. A\&A, submitted

[24] Evans II, N. J., Dunham, M. M., Jørgensen, J. K., et al. 2009, ApJS, 181, 321

[25] Lawrence, C. \& Keene, J. 2004, Spitzer Proposal, 94

[26] Stutz, A. M., Rieke, G. H., Bieging, J. H., et al. 2009, ApJ, 707, 137

[27] Paladini, R., Pagani, L., Steinacker, J., et al., 2011, Hunting Coreshine Survey, Spitzer Proposal ID \#80053

[28] Juvela, M., Ristorcelli, I., Montier, L. A., et al. 2010, A\&A, 518, L93

[29] Paladini, R., Lefèvre, C., Pagani, L., et al., in preparation.

[30] Lefèvre, C., Pagani, L., Juvela, M., et al. 2013, in Dust Growth in Star- \& Planet-forming environments, on-line presentations of a conference held in Heidelberg, 22-25 July 2013 
[31] Steinacker, J., Andersen, M., Thi, W.-F., \& Bacmann, A. 2014, A\&A, in press (arXiv:1402.1646)

[32] Paladini, R., Steinacker, J., Lefèvre, C., et al., 2012, Shedding light on grain growth in Galactic star forming regions, Spitzer Proposal ID \#90109

[33] Wright, E. L., Eisenhardt, P. R. M., Mainzer, A. K., et al. 2010, AJ, 140, 1868

[34] Andersen, M., Steinacker, J., Thi, W.-F., Pagani, L., Bacmann, A., Paladini, R. 2013, A\&A, 559, A60

[35] Juvela, M., Ristorcelli, I., Pagani, L., et al. 2012, A\&A, 541, 12

[36] Shibai, H. 2007, Advances in Space Research, 40, 595

[37] Young, E. T., Becklin, E. E., Marcum, P. M., et al. 2012, ApJ, 749, L17 